\title{
Explaining Enhanced Logical Consistency during Decision Making in Autism
}

\author{
Benedetto De Martino, ${ }^{1 \star}$ Neil A. Harrison, ${ }^{1,2 \star}$ Steven Knafo, ${ }^{2}$ Geoff Bird, ${ }^{2}$ and Raymond J. Dolan ${ }^{1}$ \\ ${ }^{1}$ Wellcome Trust Centre for Neuroimaging, University College London, London WC1N 3BG, United Kingdom, and ${ }^{2}$ Institute of Cognitive Neuroscience, \\ University College London, London WC1N 3AR, United Kingdom
}

The emotional responses elicited by the way options are framed often results in lack of logical consistency in human decision making. In this study, we investigated subjects with autism spectrum disorder (ASD) using a financial task in which the monetary prospects were presented as either loss or gain. We report both behavioral evidence that ASD subjects show a reduced susceptibility to the framing effect and psycho-physiological evidence that they fail to incorporate emotional context into the decision-making process. On this basis, we suggest that this insensitivity to contextual frame, although enhancing choice consistency in ASD, may also underpin core deficits in this disorder. These data highlight both benefits and costs arising from multiple decision processes in human cognition.

Key words: autism spectrum disorder; ASD; decision-making; rationality; economics; framing effect; skin conductance response; SCR

\section{Introduction}

Logical consistency across decisions, regardless of how choices are presented, is a central tenet of rational choice theory and the cornerstone of modern economic and political science (Luce and Raiffa, 1957; Arrow, 1982). Empirical data challenge this perspective by showing that humans are highly susceptible to the manner or context in which options are cast, resulting in a decision bias termed the "framing effect" (Tversky and Kahneman, 1974). We have previously shown that the amygdala mediates this framing bias, a finding that highlights the importance of incorporating emotional processes within models of human decision making (De Martino et al., 2006; Kahneman and Frederick, 2007). An ability to integrate emotional contextual information into the decision process provides a useful heuristic in decision making under uncertainty. This is a factor that is likely to assume considerable importance during social interactions in which information about others is often incomplete, ambiguous, and not easily amenable to standard inferential reasoning processes.

In this study, we investigated the effect of contextual frame on choice behavior of individuals with autistic spectrum disorder (ASD) (see Fig. 1). Autism is a neurodevelopmental disorder characterized by deficits in social interaction, qualitative impairments in communication, and repetitive and stereotyped patterns of behavior, interests, and activities (Kanner, 1943; Asperger, 1944). From Kanner's earliest description, it has been

\footnotetext{
Received June 23, 2008; revised July 31, 2008; accepted Aug. 4, 2008.

This work was supported by a Wellcome Trust Programme Grant (R.J.D.), a Wellcome Trust funded Clinical Research Fellowship (N.A.H.), and a Wellcome Trust PhD Scholarship (B.D.M.). We thank Uta Frith, Catherine Sebastian, and Hugo Critchley.

${ }^{*}$ B.D.M. and N.A.H. contributed equally to this work.

This article is freely available online through the $J$ Neurosci Open Choice option.

Correspondence should be addressed to Benedetto De Martino, Wellcome Trust Centre, University College Lon-

don, 12 Queen Square, London WC1N 3BG, UK. E-mail: b.martino@fil.ion.ucl.ac.uk.

DOI:10.1523/JNEUROSCI.2895-08.2008

Copyright $\odot 2008$ Society for Neuroscience $\quad$ 0270-6474/08/2810746-05\$15.00/0
}

recognized that individuals with ASD have a strong tendency to focus on parts rather than global aspects of objects of interest and are unable to integrate disparate information into a meaningful whole (weak central coherence theory) (Frith and Happé, 1994; Happé and Booth, 2008).

We previously proposed that susceptibility to a framing bias reflects the operation of an affect heuristic (De Martino et al., 2006; Kahneman and Frederick, 2007). Here, we show that individuals with ASD, a condition characterized by marked behavioral inflexibility, demonstrate a decreased susceptibility to framing resulting in an unusual enhancement in logical consistency that is paradoxically more in line with the normative prescriptions of rationality at the core of the current economics theory (Luce and Raiffa, 1957; Arrow, 1982). Furthermore, insensitivity in these subjects to a contextual framing bias was associated with a failure to express a differential autonomic response to contextual cues as indexed in skin conductance responses (SCRs), a standard measure of emotional processing. Our findings suggest that a more consistent pattern of choice in the ASD group reflects a failure to incorporate emotional cues into the decision process, an enhanced economic "rationality" that may come at a cost of reduced behavioral flexibility.

\section{Materials and Methods}

Subjects. Thirty-two subjects participated to the study, with 29 included in the final analysis. Of these 32 , three subjects were excluded: one subject was found to be on antipsychotic drug treatment (Olanzapine) after enrolment, one subject was unable to fully understand the task instructions, and a third was excluded because he was a statistical outlier (his score on the framing effect was greater than two SD above the group mean). Therefore, after exclusion, a total of 29 subjects were included in the final analysis. Fourteen ASD subjects (10 male; mean age $34.8 \pm 7.9$ years) were age and intelligence quotient (IQ) matched with 15 controls (11 male; mean age $32.2 \pm 8.5$ years). Of note, two subjects in the ASD group were taking a selective serotonin reuptake inhibitor (SSRI) antidepressant medication. These subjects were not excluded as we did not 
Table 1. Details of participants

\begin{tabular}{|c|c|c|c|c|c|c|c|c|c|c|}
\hline Sub\# & Group & Age & Gender & $\mathrm{V}-\mathrm{IQ}$ & P-IQ & $\mathrm{F}-\mathrm{IQ}$ & AD0S score1 & ADOS score2 & ADOS total score & ADOS classification \\
\hline 1 & ASD & 52.77 & $M$ & 134 & 122 & 132 & 3 & 4 & 7 & Autism spectrum \\
\hline $2^{*}$ & ASD & 28.23 & $M$ & 105 & 90 & 99 & 4 & 7 & 11 & Autism spectrum \\
\hline 3 & ASD & 29.85 & $\mathrm{~F}$ & 104 & 100 & 103 & 4 & 5 & 9 & Autism spectrum \\
\hline 4 & ASD & 30.42 & $\mathrm{~F}$ & 100 & 80 & 91 & 5 & 12 & 17 & Autism \\
\hline 5 & ASD & 22.86 & M & 112 & 99 & 107 & 4 & 8 & 12 & Autism \\
\hline 6 & ASD & 32.7 & $M$ & 119 & 125 & 124 & 3 & 7 & 10 & Autism spectrum \\
\hline 7 & ASD & 42.43 & M & 123 & 107 & 117 & 4 & 4 & 8 & Autism spectrum \\
\hline 8 & ASD & 39.13 & M & 95 & 104 & 99 & 4 & 6 & 10 & Autism spectrum \\
\hline 9 & ASD & 34.86 & M & 100 & 102 & 101 & 2 & 6 & 8 & Autism spectrum \\
\hline 10 & ASD & 29.62 & M & 125 & 119 & 125 & 3 & 4 & 7 & Autism spectrum \\
\hline 11 & ASD & 35.64 & $\mathrm{~F}$ & 114 & 116 & 116 & 4 & 10 & 14 & Autism \\
\hline 12 & $C$ & 24.17 & $\mathrm{~F}$ & 105 & 113 & 109 & & & & \\
\hline 13 & $C$ & 25.76 & M & 134 & 114 & 128 & & & & \\
\hline 14 & $C$ & 32.46 & M & 103 & 117 & 110 & & & & \\
\hline $15^{a}$ & ASD & 28.59 & M & 112 & 89 & 102 & 4 & 6 & 10 & Autism spectrum \\
\hline 16 & ASD & 24.7 & M & 124 & 125 & 128 & 3 & 6 & 9 & Autism spectrum \\
\hline 17 & $C$ & 38.68 & M & 112 & 111 & 112 & & & & \\
\hline 18 & $C$ & 53.14 & M & 105 & 113 & 109 & & & & \\
\hline 19 & ASD & 30.15 & M & 102 & 84 & 94 & 2 & 5 & 7 & Autism spectrum \\
\hline 20 & $C$ & 32.25 & $\mathrm{~F}$ & 114 & 110 & 113 & & & & \\
\hline $21^{a}$ & $C$ & 41.36 & M & 91 & 102 & 95 & & & & \\
\hline 22 & $C$ & 23.54 & M & 125 & 136 & 134 & & & & \\
\hline 23 & $C$ & 27.87 & M & 122 & 121 & 123 & & & & \\
\hline 24 & ASD & 44 & M & 112 & 107 & 110 & 1 & 2 & 3 & None \\
\hline 25 & $C$ & 24.79 & M & 111 & 111 & 112 & & & & \\
\hline 26 & ASD & 38 & $\mathrm{~F}$ & 112 & 136 & 123 & 3 & 8 & 11 & Autism spectrum \\
\hline 27 & $C$ & 28.21 & $F$ & 108 & 105 & 107 & & & & \\
\hline 28 & $C$ & 29.2 & M & 120 & 110 & 118 & & & & \\
\hline 29 & $C$ & 30.22 & M & 112 & 130 & 122 & & & & \\
\hline 30 & $C$ & 40.84 & M & 122 & 109 & 117 & & & & \\
\hline 31 & $C$ & 43.45 & $\mathrm{~F}$ & 111 & 110 & 111 & & & & \\
\hline 32 & $C$ & 28.66 & M & 118 & 124 & 122 & & & & \\
\hline
\end{tabular}

C, Control; V-IQ, verbal-IQ score; P-IQ, performance IQ score; F-IQ, full-scale IQ score; $M$, male; F, female; ADOS score 1, communication score; ADOS score 2 , reciprocal interaction score. ${ }^{a}$ Excluded (see Materials and Methods).

initially anticipate SSRIs to interfere with the cognitive abilities involved in the task; however, because it has been reported that serotonin can effect emotional processing (Canli and Lesch, 2007); we also repeated our statistical comparison excluding these two subjects (see Results). All ASD subjects were diagnosed using international classification of diseases-10 (ICD-10) criteria by specialized clinicians before inclusion (Table 1). An additional subject (ASD group) was excluded from the SCR analysis, attributable to technical failure. Subjects performed a framing financial decision making task (see below, Experimental paradigm), previously described in De Martino et al. (2006). SCR was recorded throughout data acquisition.

The ASD group, comprising 10 males and 4 females, had a mean age of 34.8 years (SD, \pm 7.9 years) and a mean full-scale IQ score of $112.1 \pm 13.5$ (mean verbal IQ score, $112.6 \pm 11.6$; and mean performance IQ score, $109.0 \pm 16.1)$. All ASD subjects had received a clinical diagnosis of autism or ASD by a specialized clinician before inclusion. In addition, all subjects had an independent autism diagnostic observation schedule (ADOS) assessment to quantify the degree of impairment across the domains of communication and reciprocal social interaction (Lord et al., 2000). The ADOS is a research standardized test that provides an index of autistic symptoms observed on a particular occasion. All subjects took module 4 (designed to assess adults) and received two scores: (1) a communication score (labeled "ADOS score 1" in Table 1) on which the ASD cutoff is 2 and the autism cutoff is 4 and (2) a reciprocal interaction (or "social") score (labeled "ADOS score 2") on which the ASD cutoff is 4 and the autism cutoff is 7. Overall, the total ADOS score cutoff for ASD is 7 and the cutoff for autism is 12 . We note, however, that the ADOS classification is not a clinical diagnosis (i.e., based on standardized DSMIV/ICD-10) (Table 1) and, although it may contribute to diagnosis, cannot replace full systematic assessment by a specialized clinician. For this reason, one ASD subject (subject 24) had a clinical diagnosis of autism, although scored below the ADOS cutoff for autism spectrum disorder.

The control group, comprising 11 males and 4 females, had a mean age of $32.2 \pm 8.5$ and mean full-scale IQ score of $116.5 \pm 7.7$ (mean verbal IQ score, $114.8 \pm 8.5$; mean performance IQ score, $115.6 \pm 8.5)$. There were no significant differences between the two groups in age $(p=0.41$, independent $t$ test), full-scale IQ score ( $p=0.29$, independent $t$ test), verbal IQ score ( $p=0.56$, independent $t$ test), or performance IQ score ( $p=0.17$, independent $t$ test) (Table 1 ).

The study was conducted with the approval of the National Hospital for Neurology and Neurosurgery and the Institute of Neurology Joint Research Ethics Committee, London, UK.

Experimental paradigm. The experiment was divided into three parts: an instruction phase, a task phase during which subjects performed the task (during this phase SCR was recorded), and a questionnaire phase. In the instruction phase, subjects were familiarized with the decisionmaking task and given a number of practice trials before starting the main behavioral task.

The task was divided into three 17 min sessions. Each session comprised 96 trials ( 32 loss frame, 32 gain frame, and 32 catch trials) ordered pseudorandomly. At the beginning of each trial, participants were shown a message indicating the initial amount of money that they received to play that trial (e.g., "you receive $\mathfrak{E 5 0 " ) ~ ( 2 ~ s ) . ~ F o u r ~ d i f f e r e n t ~ s t a r t i n g ~}$ amounts were used in the experiment: $25,50,75$, and $£ 100$. Participants were instructed that they would not be able to retain the whole of this initial amount but would have to choose between a sure and a gamble (risky) option (4s).

In the gain frame trials, the sure option was presented as the amount of

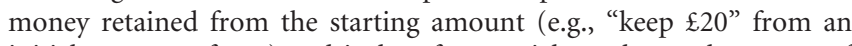
initial amount of $\mathfrak{E 5 0}$ ) and in loss frame trials as the total amount of 


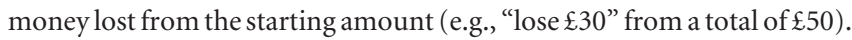
The gamble option was identical for both frames and was represented by a pie chart depicting the probability of winning and losing in green and red, respectively (Fig. 1). Four different probabilities were used in the experiment: $20,40,60$, and $80 \%$. All experimental variables (total starting amount, percentage of the money offered, number of trials per session) were fully counterbalanced between frame conditions. The expected value of the options were balanced in each trial (with the exception of the catch trials, see below) and mathematically equivalent between frames. For example, if participants initially received $\mathfrak{£} 50$, they were then required to choose between the options "keep $£ 20$ " or a gamble with a $40 \%$ chance of winning $\mathfrak{E} 50$ and a $60 \%$ chance of winning nothing. Participants were required to respond within $4 \mathrm{~s}$ by key press.

Given the equivalence of the choices in terms of expected outcomes, we included "catch" trials ( 32 trials each session) to ensure that subjects remained actively engaged in the decision-making task throughout the course of the experiment. In these catch trials, in both frames, expected outcomes for the sure and gamble option were markedly unbalanced: in half of the trials ("gamble weighted") the gamble option was preferable (e.g., $95 \%$ probability of winning by taking the gamble option vs a sure choice of $50 \%$ of the initial amount), and for the other half of trials ("sure weighted") the sure option was preferable (e.g., $5 \%$ probability of winning by taking the gamble option vs a sure choice of $50 \%$ of the initial amount). As in the main experimental trials, the catch trials were also presented in either a gain or a loss frame.

Note that the behavioral task they were asked to perform was identical to the one used in the functional magnetic resonance imaging (fMRI) experiment described by De Martino et al. (2006).

Behavioral data analysis. The behavioral data were analyzed using the statistic software SPSS. The main effect of frame was calculated using a mixed measures ANOVA with frame (gain and loss) as the within subject factor and group (ASD and control) as the between subject factor. Significant main effects and interactions were then further analyzed using paired and independent sample $t$ tests. Effect of frame within each group (i.e., ASD and control) was calculated as the percentage of trials in which subjects chose the "gamble" option within each frame. The incorrect catch trials responses (i.e., when the subject fails to chose the option with the dominant expected value independently of the framing manipulation) were analyzed using a two-sample (i.e., ASD and control) $t$ test.

SCR data analysis. SCR was recorded using two $\mathrm{Ag} / \mathrm{AgCl}$ electrodes (skin conductance recorder, AT-64; Autogenic Systems Laboratory) attached using surgical tape to the palmar surface of subjects distal phalanges of the second and fourth fingers of the left hand (Dawson et al., 2000). Conductive gel was applied between the skin and the electrodes. SCR signal and event markers were sent to an analog-to-digital converter (CED-Power1401) then recorded on two different channels using the software Spike2 (v5; Cambridge Electronic Design).

Skin conductance responses and event markers were recorded at 100 Hz. The window of interest was defined as the $5 \mathrm{~s}$ after the subject's decision. Baseline skin conductance was calculated by averaging the skin conductance level during the $0.5 \mathrm{~s}$ before the presentation of the task. Amplitude of the SCR was defined as the difference between the timewindow peak value and the baseline. Attributable to technical failure, skin conductance for one subject (ASD) was not recorded, and three further subjects (total of two from each group) were subsequently excluded because of lack of physiological SCR responses [amplitude $<2$ microSiemens $(\mu \mathrm{S})]$. All remaining SCR data were visually inspected and nonphysiological SCR responses ( $\angle 10 \%$ of the entire data set) were removed before further analysis. SCR responses to each trial were recorded for each subject. Mean SCR and SD of SCR responses were then calculated and used to produce $z$-transformed SCR responses for each trial per subject (Yaremko, 1986)

$Z$-transformed SCR results were then analyzed using a $2 \times 2 \times 2$ mixed-measures ANOVA with within-subject factors of frame (loss frame, gain frame) and choice (risky choice, sure choice), group (ASD, control) was the between-subject factor.
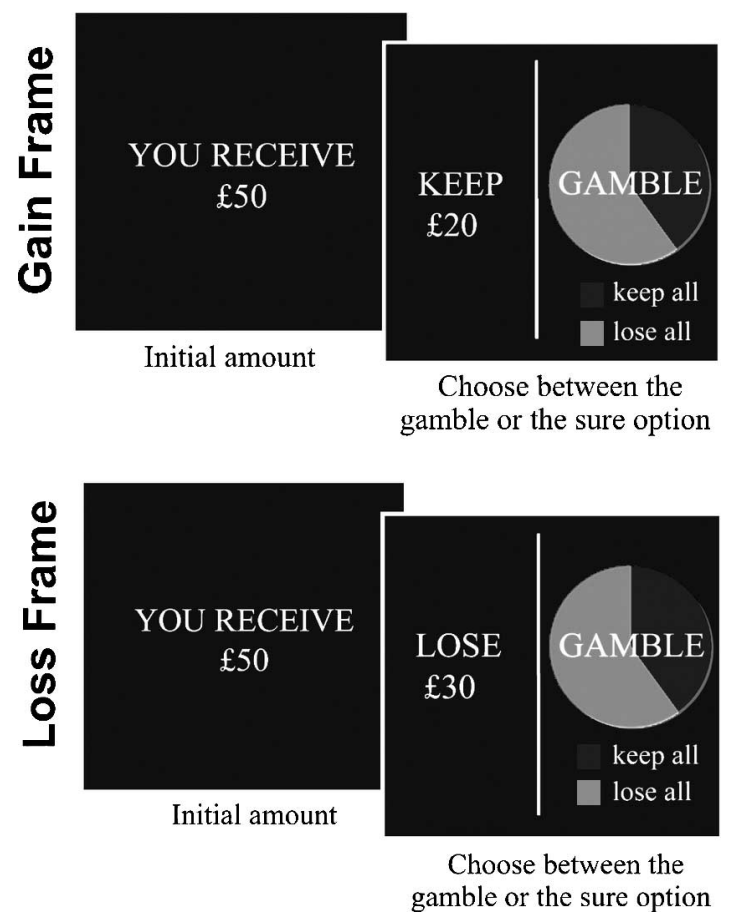

Figure 1. Task. Participants were shown a message indicating the amount of money received to play in that trial (e.g., "you receive $f 50$ "). Subjects then had to choose between a "sure" or a "gamble" option presented in the context of two different "frames." The "sure" option was formulated as either the amount of money retained from the initial starting amount (e.g., keep $£ 20$ of a total of $£ 50$-gain frame) or as the amount of money lost from the initial amount (e.g., lose $£ 30$ of a total of $£ 50$-loss frame). The "gamble" option was identical in both frames and represented as a pie chart depicting the probability of winning or losing.

\section{Results}

In accordance with our previously reported findings, all control subjects manifest susceptibility to the framing effect, reflected in an increased disposition to gamble in loss compared with gain frames (mean, $14.24 \pm 1.68 \%$ ). This susceptibility to the frame manipulation was markedly reduced in the ASD group (mean, $7.66 \pm 1.95 \%$ ) (Fig. $2 A$ ). The significance of this effect was confirmed in an interaction analysis showing that the ASD subjects were significantly more consistent (i.e., less influenced by the frame) in their pattern of choice compared with controls [ANOVA; group (ASD, control) $\times$ frame (gain, loss); $F_{(1,27)}=$ 6.56, $p<0.02]$.

The ASD group also showed mild risk aversion compared with controls evidenced in a trend level main effect of group $\left(F_{(1,27)}=\right.$ 3.91, $p=0.058$ ) (Fig. $2 B$ ). To ensure that our findings were not driven by greater risk aversion in the ASD group, we repeated the ANOVA with individual risk tendency (calculated as percentage of risky choices independently of frame) as a covariate. This analysis corroborated our previous findings [ANOVA; group (ASD, control) $\times$ frame (gain, loss); $\left.F_{(1,26)}=10.03, p<0.005\right]$ by showing that the ASD group was significantly less affected by the framing decision bias even after controlling for differences in absolute risk attitude. This result held even after reducing the size of the ASD group (see Materials and Methods) by excluding the two ASD subjects taking SSRI medication [ANOVA; group $\left(\mathrm{ASD}\right.$, control) $\times$ frame (gain, loss); $\left.F_{(1,25)}=5.04, p<0.02\right]$.

To control that the ASD subjects were fully engaged in the task and were responding to monetary incentives similarly to the control group, we analyzed the performance in the catch trials response (see Materials and Methods) by two-tailed paired $t$ test. 


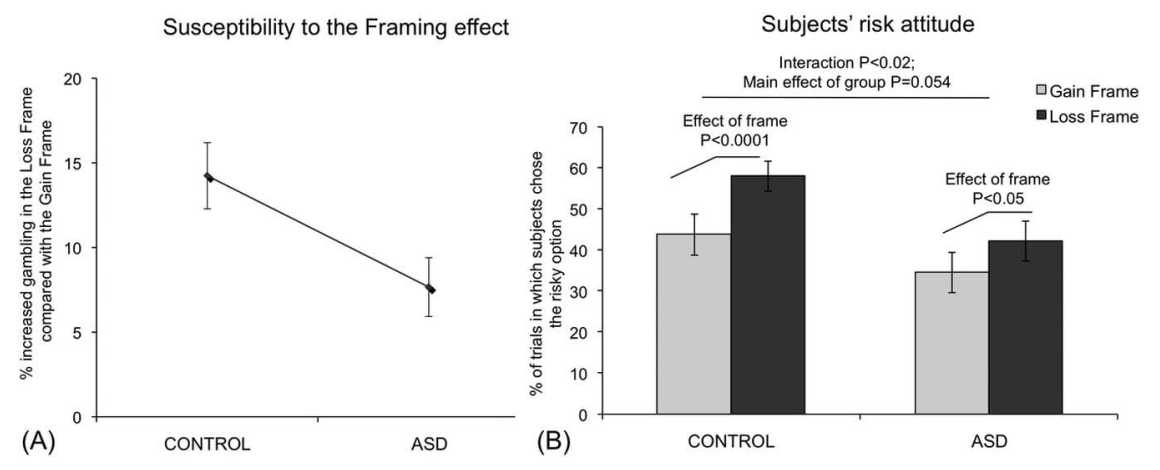

Figure 2. Behavioral data. $\boldsymbol{A}$, Summary of the susceptibility of subjects to the framing manipulation (mean $\pm \mathrm{SE}$ ) calculated as a percentage of increased gambling in the loss frame compared with the gain frame. The susceptibility to the framing manipulation is significantly reduced in the ASD group (mean, $7.66 \pm 1.95 \%$ ) compared with the control group (mean, $14.24 \pm 1.68 \%$ ) [ANOVA; group (ASD, control) $\times$ frame (gain, loss); $F_{(1,27)}=10.03, p<0.005$ ]. $\boldsymbol{B}$, Percentage of trials in which subjects chose the risky option rather than the sure option for both frame manipulations: in the control group, subjects gambled in $43.75 \%$ ( \pm 4.72 ) of the trials in the gain frame and $57.99 \%$ ( \pm 4.70 ) of the trials in the loss frame whereas in the ASD group the percentages were respectively $34.52 \%$ ( \pm 4.99 ) and $42.19 \%$ ( \pm 3.64$)$. A group (ASD, control) $\times$ frame (gain, loss) $2 \times 2$ ANOVA yielded a highly significant main effect of frame $\left(F_{(1,27)}=72.83, p<0.0001\right)$ in accordance with framing effect manipulation (increase in risk in loss frame compared with the gain frame). The critical two-way interaction (group, frame) yielded a significant result $\left(F_{(1,27)}=\right.$ $6.56, p<0.02$ ), supporting our hypothesis that ASD subjects have a significantly reduced susceptibility to the framing manipulation compared with the control group. Notably, the ASD group showed a mild risk aversion compared with controls evidenced by trend level main effect of group $\left(F_{(1,27)}=3.91, p=0.058\right)$.

Effects of Frames on mean SCR Response

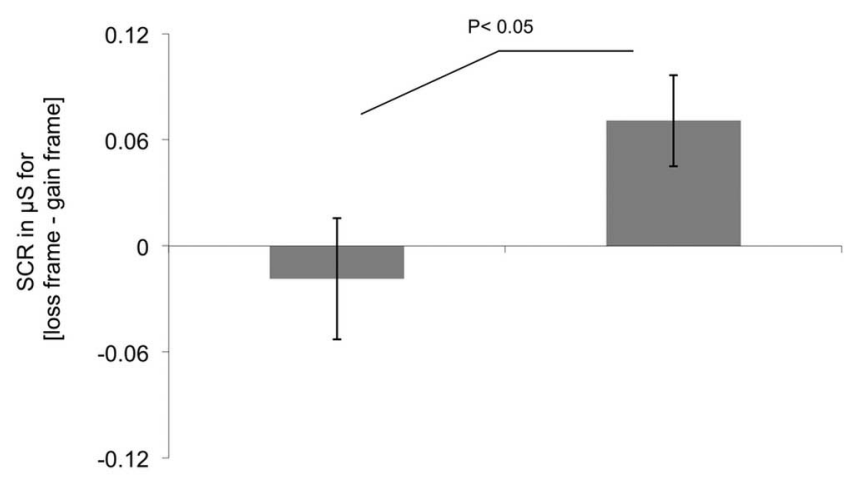

ASD

CONTROL

Figure 3. SCR data. Mean difference in $S C R$ amplitude in $\mu S$ to the loss versus gain frame in each group. Control group $(0.071 \mu \mathrm{S} ; \pm 0.026 \mu \mathrm{S})$ and ASD group $(-0.019 \mu \mathrm{S} ; \pm 0.034 \mu \mathrm{S})$ showing loss of differential SCR response to the framing manipulation in the ASD group compared with controls [ANOVA (group, frame, choice); $F_{(1,23)}=4.86, p<0.05$ ].

This statistical comparison did not show significant difference between the two groups $\left(t_{(27)}=1.8, p=0.09\right)$. Furthermore, in light of a possible trend level effect, we additionally ensured that the individual performance on catch trials did not impact on the main conclusion by repeating our critical statistical comparison [ANOVA; group (ASD, control) $\times$ frame (gain, loss); $F_{(1,27)}=$ 7.23, $p<0.02$ ] covarying out individual performance on the catch trials; as can be seen, our main effect remained significant.

Acquisition of subjects' SCRs, while they performed the task, enabled us to obtain an on-line measurement of their autonomic responses to the framing manipulation. Analysis of these data revealed that the ASD group showed a higher absolute SCR response for each frame (gain frame, $0.72 \pm 0.14 \mu \mathrm{S}$; loss frame, $0.70 \pm 0.12 \mu \mathrm{S}$ ) compared with the control group (gain frame, $0.47 \pm 0.15 \mu \mathrm{S}$; loss frame, $0.54 \pm 0.18 \mu \mathrm{S}$ ), probably attributable to increased anxiety. However, the ASD individuals critically showed a lack of a differential response to the frame manipulation. In fact, although loss and gain frames induced differential skin conductance responses in controls $\left(t_{(12)}=3.19, p<0.01\right.$, paired $t$ test $)$, SCR did not differentiate between frames in the ASD group $\left(t_{(11)}=0.96, p=0.36\right.$, paired $t$ test) (Fig. 3). These results were again corroborated by a significant two-way interaction (frame; group; $F_{(1,23)}=4.86, p<$ $0.05)$ but no main effect of group $\left(F_{(1,23)}=\right.$ $0.8, p=0.46)$ in a $2 \times 2 \times 2$ ANOVA (group, frame, choice). These data further support our contention that ASD subjects, although having an absolute emotional response, crucially fail to differentially engage emotional processes in response to the framing manipulation.

\section{Discussion}

These findings suggest the ASD group fail to integrate emotional contextual cues into the decision-making process. This is evident both in a reduced behavioral susceptibility of a framing effect and an absence of a differential SCR response to our contextual manipulation. The concept that ASD individuals fail to integrate information across cognitive domains also informs the suggestion that an uneven profile of abilities and deficiencies in autistic individuals may reveal an imbalance in empathizing and systemizing behaviors (Baron-Cohen and Belmonte, 2005).

Our SCR results in the context of a high-level decision task supports convergent evidence of impairment in emotional processing in autism (Baron-Cohen et al., 2000; Adolphs, 2002). Paradoxically, although this impairment in processing contextual emotional information protects ASD subjects from the framing bias, leading to more consistent behavior in situations of risk, it may come at a cost of the social, emotional, and behavioral deficits that characterize the condition.

Recent theoretical accounts of decision making have put forward a "two-systems" model of human judgment (Evans, 2003). This view proposes that human decision making arises through a combination of intuitive and analytic processes. This model proposes that intuitive reasoning is rapid and capable of processing large amounts of information in parallel; however, it is prone to mistakes and strongly influenced by contextual emotional information (Kahneman, 2003). In contrast, analytical reasoning is more accurate but slow and computationally demanding. According to this view, the framing bias reflects an affect heuristic by which normal individuals incorporate a potentially broad range of additional emotional information into the decision process. In evolutionary terms, this mechanism may confer a strong advantage because such contextual cues may carry useful, even critical, information that dictates a rapid response. We propose that this ability is particularly crucial in a social context in that subtle contextual cues communicate knowledge elements (possibly unconscious) that allow optimal decisions to be made in uncertain environments (Stanovich and West, 2002).

In the context of the "two-systems" model of decision making described above, these results suggest that ASD individuals have an increased tendency toward the analytic type of decision making, attributable to impairment within their intuitive reasoning mechanisms. This interpretation would also support the 
empathizing-systemizing (E-S) theory of autism (Baron-Cohen and Belmonte, 2005). The E-S theory proposes that the imbalance between analytic and empathic behavior underlies both the impairment in social skills in ASD and their enhanced analytical skills. During the framing task, ASD subjects were better able to ignore biasing contextual information and isolate the critical information about the numerical value of the sure and risky options. This result is consistent with other experimental findings showing that ASD have enhanced attention for the task's details but reduced capacity to deal with the global aspect of the task as predicted by weak coherence theory (Frith and Happé, 1994).

In a previous fMRI study (De Martino et al., 2006; Kahneman and Frederick, 2007), we showed that the engagement of an amygdala-based emotional system played a key role in underpinning a framing effect. These previous results, combined with the SCR data shown here, suggest that the failure to assign emotional salience to contextual cues and consequential lack of behavioral bias in ASD may result from an amygdala based mechanism. A wealth of empirical data supports this hypothesis. First, histopathological abnormalities of the amygdala such as an increased cell density and a reduced dendritic arborization have been described in autism (Bauman and Kemper, 1994). Moreover, Howard et al. (2000) suggested that persons with high-functioning autism showed a similar neuropsychological profile to that seen in patients with amygdala lesions, particularly a selective impairment in the recognition of facial expressions of fear. Last, several imaging studies demonstrate blunted activation of the amygdala in ASD during tasks which involved processing of facial expressions (BaronCohen et al., 2000; Critchley et al., 2000).

An ability to incorporate a broad range of contextual cues into the decision process endows humans with a clear evolutionary advantage. This requirement becomes particularly acute when making decisions in social environments in which multiple subtle contextual cues carry critical information. We suggest that competence in dealing with social uncertainty involves deployment of affect heuristics. In ASD, a reduction of framing effect might explain both enhanced analytic and impoverished social abilities, the latter reflecting an inability to deploy an affect heuristic in complex and uncertain social contexts. Thus, our findings suggest a potential core neurobiological deficit in ASD and also speak to limits, and trade-offs, in human rationality.

\section{References}

Adolphs R (2002) Neural systems for recognizing emotion. Curr Opin Neurobiol 12:169-177.
Arrow KJ (1982) Risk perception in psychology and economics. Econ Inq 20:1-9.

Asperger H (1944) Die „Autistischen Psychopathen“ im Kindesalter. Eur Arch Psychiatry Clin Neurosci 117:76-136.

Baron-Cohen S, Belmonte MK (2005) Autism: a window onto the development of the social and the analytic brain. Annu Rev Neurosci 28:109-126.

Baron-Cohen S, Ring HA, Bullmore ET, Wheelwright S, Ashwin C, Williams SCR (2000) The amygdala theory of autism. Neurosci Biobehav Rev 24:355-364.

Bauman ML, Kemper TL (1994) Neuroanatomic observations of the brain in autism. In: The neurobiology of autism (Bauman ML, Kemper TL, eds), pp 119-145. Baltimore: Johns Hopkins UP.

Canli T, Lesch K, (2007) Long story short: the serotonin transporter in emotion regulation and social cognition. Nat Neurosci 10:1103-1109.

Critchley HD, Daly EM, Bullmore ET, Williams SC, Van Amelsvoort T, Robertson DM, Rowe A, Phillips M, McAlonan G, Howlin P, Murphy DG (2000) The functional neuroanatomy of social behavior. Brain 123:2203-2212.

Dawson ME, Schell AM, Filion DL (2000) The electrodermal system. In: Handbook of psychophysiology, Ed 2 (Cacioppo J, Tassinary LG, Berntson GG, eds), pp 200-223. Cambridge, UK: Cambridge UP.

De Martino B, Kumaran D, Seymour B, Dolan RJ (2006) Frames, biases, and rational decision-making in the human brain. Science 313:684-687.

Evans JS (2003) In two minds: dual-process accounts of reasoning. Trends Cogn Sci 7:454-459.

Frith U, Happé F (1994) Autism: Beyond "theory of mind." Cognition 50:115-132.

Happé FG, Booth RD (2008) The power of positive: revisiting weak coherence in autism spectrum disorders. Q J Exp Psychol 61:50-63.

Howard MA, Cowell PE, Boucher J, Broks P, Mayes A, Farrant A, Roberts N (2000) Convergent neuroanatomical and behavioural evidence of an amygdala hypothesis of autism. Neuroreport 11:2931-2935.

Kahneman D (2003) Maps of bounded rationality: psychology for behavioral economics. Am Econ Rev 93:1449-1475.

Kahneman D, Frederick S (2007) Frames and brains: elicitation and control of response tendencies. Trends Cogn Sci 11:45-46.

Kanner L (1943) Autistic disturbances of affective contact. Nerv Child 2: 217-250.

Lord C, Risi S, Lambrecht L, Cook EH, Leventhal BL, DiLavore PC, Pickles A, Rutter M (2000) The Autism diagnostic observation schedule-Generic: a standard measure of social and communication deficits associated with the spectrum of autism. J Autism Dev Disord 30:205-223.

Luce RD, Raiffa H (1957) Games and decisions. New York: Wiley.

Stanovich K, West F (2002) Individual differences in reasoning: implications for the rationality debate. In: Heuristic and biases: the psychology of intuitive judgement (Gilovich T, Griffin D, Kahneman D, eds), pp 421440. Cambridge, UK: Cambridge UP.

Tversky A, Kahneman D (1974) Judgment under uncertainty: heuristics and biases. Science 185:1124-1131. 